\title{
OBSERVATIONS OF SMALL-SCALE ENERGETIC EVENTS IN THE SOLAR TRANSITION REGION: EXPLOSIVE EVENTS, UV BURSTS, AND NETWORK JETS
}

\author{
Zhenghua Huang \\ Institute of Space Sciences, Shandong University, \\ Weihai, Shandong, China, z.huang@sdu.edu.cn
}

\section{Bo Li}

Institute of Space Sciences, Shandong University, Weihai, Shandong, China, bbl@sdu.edu.cn

\author{
Lidong Xia \\ Institute of Space Sciences, Shandong University, \\ Weihai, Shandong, China, xld@sdu.edu.cn
}

\begin{abstract}
In this paper, we review observational aspects of three common small-scale energetic events in the solar transition region (TR), namely TR explosive events, ultraviolet bursts and jets. These events are defined in either (both) spectral or (and) imaging data. The development of multiple instruments capable of observing the TR has allowed researchers to gain numerous insights into these phenomena in recent years. These events have provided a proxy to study how mass and energy are transported between the solar chromosphere and the corona. As the physical mechanisms responsible for these small-scale events might be similar to the mechanisms responsible for large-scale phenomena,
\end{abstract}

such as flares and coronal mass ejections, analysis of these events could also help our understanding of the solar atmosphere from small to large scales. The observations of these small-scale energetic events demonstrate that the TR is extremely dynamic and is a crucial layer in the solar atmosphere between the chromosphere and the corona.

Keywords: transition region, magnetic reconnection, small-scale dynamics, spectroscopic observations

\section{THE TRANSITION REGION OF THE SOLAR ATMOSPHERE}

The transition region (TR) of the solar atmosphere is a region between the solar chromosphere and corona, which is assumed to be a thin layer (a few hundred kilometers) in one-dimensional static models of the solar atmosphere (e.g. [Vernazza et al., 1981; Avrett, Loeser, 2008]. Across this region, the temperature of the solar atmosphere rises from about $2 \cdot 10^{4} \mathrm{~K}$ in the upper chromosphere to about $10^{6} \mathrm{~K}$ in the corona [Mariska, 1992]. As a critical region between the chromosphere and corona, TR holds the key to understanding how energy and mass are transported from the lower to higher solar atmosphere. It therefore attracts a lot of attention of the community. Recent observational and theoretical studies have revealed that the TR is highly dynamic and nonuniform. Figure 1 displays schematic structures of the lower solar atmosphere, which presents dynamics and complexity in the region. Thus, the TR is often regarded as a temperature region rather than a real geometrical layer. More details (both historical and recent advance) of the TR could be found in review articles [Mariska, 1986, 1992; Tian, 2017; Young et al., 2018].

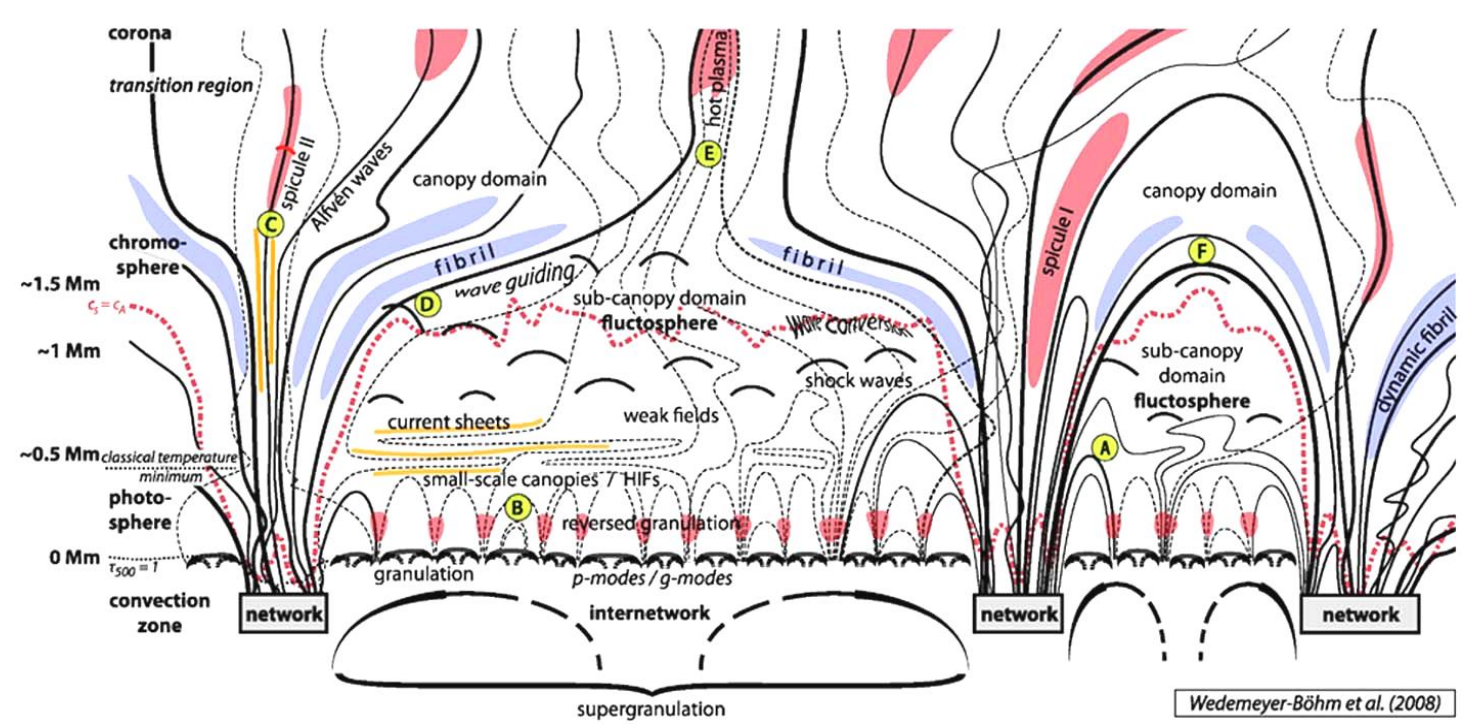

Figure 1. Schematic structures of the lower solar atmosphere (adapted from [Wedemeyer-Böhm, 2009]) 
TR temperatures show that the TR mostly emits in far ultraviolet (FUV) especially by ions such as C IV, O IV and Si IV. Thus, observations of the solar transition region became available only in the space era. The $\mathrm{Na}$ val Research Laboratory's High Resolution Telescope and Spectrograph (HRTS) [Bartoe, Brueckner, 1975] recorded ultraviolet spectra of the Sun during 10 shuttle flights, which revealed many dynamic phenomena in the spectral lines formed in the TR (see e.g. [Brueckner, Bartoe 1983; Dere et al., 1989, 1991]. Later in the 1990s, the Solar and Heliospheric Observatory (SOHO) [Domingo et al., 1995] was launched carrying many instruments including two that could observe the TR, the Solar Ultraviolet Measurements of Emitted Radiation (SUMER) [Wilhelm et al., 1995; Lemaire et al., 1997] and the Coronal Diagnostic Spectrometer (CDS) [Harrison et al., 1995]. SUMER provided full wavelength spectra of the Sun from 500 to $1610 \AA$, which have formation temperatures from $10^{4}$ to $10^{6} \mathrm{~K}$. SUMER is a powerful tool for studies of the TR, and has revealed various small-scale structures of the TR in terms of intensity, velocity, density, and temperature (see, e.g., [Hassler et al., 1999; Xia et al., 2003, 2004; Tu et al., 2005]. CDS is designed to detect solar extreme ultraviolet radiation that allows us to examine conditions in the solar corona. The spectra recorded by CDS also include a few TR lines that help to discover some small-scale dynamics such as blinkers [Harrison, 1997] in the region. The Transition Region and Coronal Explorer (TRACE) [Handy et al., 1999], launched in late 1990s, provided us with high spatial-temporal resolution images of the solar atmosphere that allowed us to follow the evolution of the dynamic phenomena in the TR. A few more satellites aiming at studying the Sun were launched in the first 20 years of the $21^{\text {st }}$ century. An important mission is Hinode [Kosugi et al., 2007] launched in 2006. Hinode carries three instruments: the Extreme Ultraviolet Imaging Spectrometer (EIS) [Culhane et al., 2007], Solar Optical Telescope (SOT) [Tsuneta et al., 2008], and X-Ray Telescope (XRT) [Golub et al., 2007], which could take spectra, X-ray images, and magnetic field data of the Sun. These data could help understand the connection among activities seen in different temperatures from that in the photosphere to that in the corona. Another very successful mission to study the Sun is the Solar Dynamics Observatory (SDO) [Pesnell et al., 2012] launched in 2010. Aboard SDO there are two instruments: the Atmospheric Imaging Assembly (AIA) [Lemen et al., 2012] and the Helioseismic and Magnetic Imager (HMI) [Scherrer et al., 2012], which take high spatial-temporal resolution images of the full-disk. AIA takes continuous full-disk observations of the solar chromosphere and corona in seven extreme ultraviolet (EUV) channels, spanning a temperature range from approximately $20 \cdot 10^{3}$ to $20 \cdot 10^{6} \mathrm{~K}$. HMI providing full-disk magnetic field observations of the Sun, these data help us to link different dynamics in the solar atmosphere from temperature to temperature and from location to location. The Interface Region Imaging Spectrograph (IRIS) [De Pontieu et al., 2014b] was launched in 2013. It is designed to study the solar chromosphere and TR, and it provides fruitful observa- tions of the TR with unprecedented spatial and spectral resolutions. IRIS has the unique capability of acquiring both imaging and spectral data on the TR simultaneously, and it provides fruitful results on the dynamics of the TR (e.g. [De Pontieu et al., 2014a; Testa et al., 2014; Tian et al., 2014; Peter et al., 2014; Hansteen et al., 2014]. The above instruments have revealed the dynamic and complex nature of the TR, which presents many smallscale energetic events seen in imaging and spectroscopic data. In this paper, we review the observational facts of three types of such small-scale energetic events in the TR as transition region explosive events, UV bursts, and network jets.

\section{TRANSITION REGION EXPLOSIVE EVENTS}

In the 1970s, the Naval Research Laboratory launched three Black Brant sounding rockets with the High Resolution Telescope and Spectrograph (HRTS) experiment onboard [Brueckner, Bartoe, 1983]. With a slit 900 arcsec long and 0.5 arcsec wide, the experiments provided spectral data over a wavelength range from 1175 to $1710 \AA$ with a spectral resolution of $0.05 \AA$. The obtained spectra of Si IV $\left(8.0 \cdot 10^{4} \mathrm{~K}\right), \mathrm{C}$ IV $\left(1.0 \cdot 10^{5} \mathrm{~K}\right)$, and O IV $\left(1.3 \cdot 10^{5} \mathrm{~K}\right)$ emitted from the TR exhibit profiles with strong wings extended to $50-250 \mathrm{~km} / \mathrm{s}$ toward both short and long wavelengths. These events have 1-2 arcsec spatial extension along the slit, which has been taken as their spatial size. Their lifetime was however difficult to estimate because of the lack of imaging datasets. Huang et al. [2014a] have found that the brightening corresponding to a TREE could last for about one hour. Brueckner and Bartoe [1983] proposed that such spectra are emitted from turbulent events. While Dere et al. [1984] presented their study on the TR spectral data from HRTS, they called such events explosive type of events (i.e. explosive events). The term of transition region explosive events (TREEs) started to be widely used since Dere et al. [1989] presented a statistical analysis of the HRTS data with detailed examples of such events. Figure 2 presents an example of TREE spectrum observed by IRIS. Although TREEs are mostly identified in the TR emissions, some of them can also produce signatures in spectra from lower temperatures, such as C I, C II, O I, Lyman series, Mg II, etc (see, e.g., [Dere 1992; Madjarska, Doyle, 2002; Zhang et al., 2010; Huang et al., 2014b].

According to the Doppler effect, the extended strong wings in the TR spectra indicate the existence of opposite-directional plasma flows in the source. Therefore, TREEs have also been known as "bi-directional jets". Such Doppler velocities can be generated by any mechanisms that can produce bi-directional flows within the space of the pixel size. For example, swirling jets suggest plasma motion along magnetic field lines [Curdt, Tian, 2011] and bi-directional flows along two close flux tubes [Alexander et al., 2013]. Because TREEs normally also exhibit an enhancement of radiation, their observations also match the picture of magnetic reconnection, which predicts bi-directional outflows and energy 

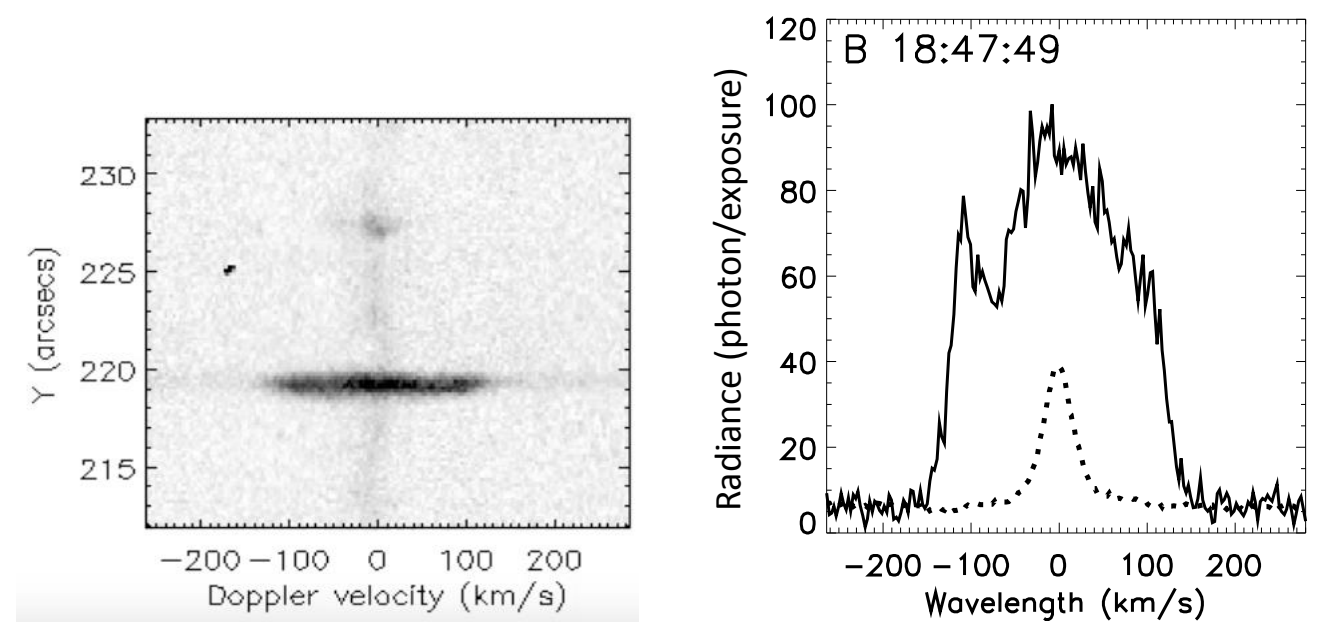

Figure 2. An example of Si IV spectral image of a transition region explosive event. The observations were taken by IRIS on June 27, 2013 as shown in Figure 5. Left panel - the event when it was crossing the slit. The image is shown in reversedcolor scale, which presents strong emission in black and weak in white. The explosive event is presented at the position $Y=219 \div 220$ arcsec. Right panel — the spectrum of the event (solid line). The normal spectrum is shown by dashed line (adapted from [Huang et al., 2014a]

release (see [Priest, Forbes, 2000; Priest, 2014] for theory and [Su et al., 2013; Yang et al., 2015; Sun et al., 2015; Li et al., 2016, 2018a; Xue et al., 2016; Huang et al., 2018] for real examples in the solar atmosphere). With this clue, magnetic reconnection is thought to be one of the mechanisms for producing TREEs [Dere et al., 1989, 1991; Innes et al., 1997, 2015; Li, 2019], and has been evidenced by observations from instruments such as SUMER and IRIS (Innes et al., 1997; Huang et al., 2014b, 2018]. Figure 3 shows spectral examples of TREEs as seen by SUMER raster scan. It indicates that the spectra are one wing enhanced on one side, other wing enhanced on the other side, and both wing enhanced in the middle of the TREEs. These observations provide a solid evidence for the magnetic reconnection scenario as shown in Figure 4.

The magnetic reconnection scenario has been indirectly supported by observations of TREE associated phenomena. TREEs are usually related to mixed polarities of magnetic features at boundaries of supergranulation cells [Brueckner et al., 1988; Dere et al., 1989, 1991; Porter, Dere, 1991; Chae et al., 1998a; Madjarska, Doyle, 2003; Teriaca et al., 2004; Ning et al., 2004; Muglach, 2008]. Moreover, Chae et al. [1998a] have found that more than $60 \%$ of TREEs are associated with magnetic cancellation, a phenomenon usually occurring after magnetic reconnection [Zwaan 1987; van Ballegooijen, Martens, 1989; Huang et al., 2012, 2014a, b; Mou et al., 2016].

Moreover, many studies have focused on what dynamic features in the solar atmosphere can produce TREE spectra, and they have been found to be signatures of siphon-flows in small-scale loops [Teriaca et al., 2004], spicules [Wilhelm, 2000], chromospheric upflows [Chae et al., 1998b], surges [Madjarska et al., 2009], swirling jets [Curdt, Tian, 2011], and coronal brightenings and jets [Madjarska et al., 2012; Huang et al., 2012; Li et al., 2018b]. These observations suggest that TREEs can be triggered in ways other than magnetic reconnections.
Recently, the very high-resolution imaging and spectral data that are simultaneously taken by IRIS have revealed more details of TREEs. Huang et al. [2014a] studied a TREE that was associated with a dot-like brightening at a coronal-hole boundary, and they found that the TREE was associated with interactions between small-scale loops and small-scale jets (see Figure 5). The authors suggested that the interaction between loops could lead to magnetic reconnection that is responsible for the TREE. The connection between the loop-loop interaction and TREEs has also been confirmed by Huang et al. [2015]. Huang et al. [2017] have studied 24 TREEs found in active regions, aiming at finding out which phenomena in the chromosphere and transition region are associated with TREEs. They have established that 16 TREEs are associated with stationary loop-brightenings without any response in chromospheric $\mathrm{H} \alpha$ spectra; six are associated with propagating loop brightenings, in which two events are associated with dark jets observed in chromospheric $\mathrm{H} \alpha$ lines; two are associated with brightenings in the conjunction region of multiple loops seen in the transition region. The study by Huang et al. [2017] clearly indicates the close relation between TREEs and dynamics of TR loops. Since the dynamics of loops could directly link to how mass and energy are transported in the solar atmosphere [Klimchuk, 2006], the interaction between two loop systems might result in magnetic reconnection that could exchange plasma between the two systems and generate heats therein [Huang et al., 2018]. Such loop interactions might generate TREE spectra (see [Huang et al., 2015]), and thus TREE could be a signal for searching magnetic reconnection between loops in the solar atmosphere. In the other conditions, a loop system might contain multiple threads that might be braided due to the random motions in the photosphere [Parker, 1983a]. The braiding magnetic structures could build up magnetic energy via small-scale magnetic reconnection events in the solar atmosphere [Parker, 1983b, 1988; Klimchuk, 2006; Schrijver, 2007; Cirtain et al., 2013]. 


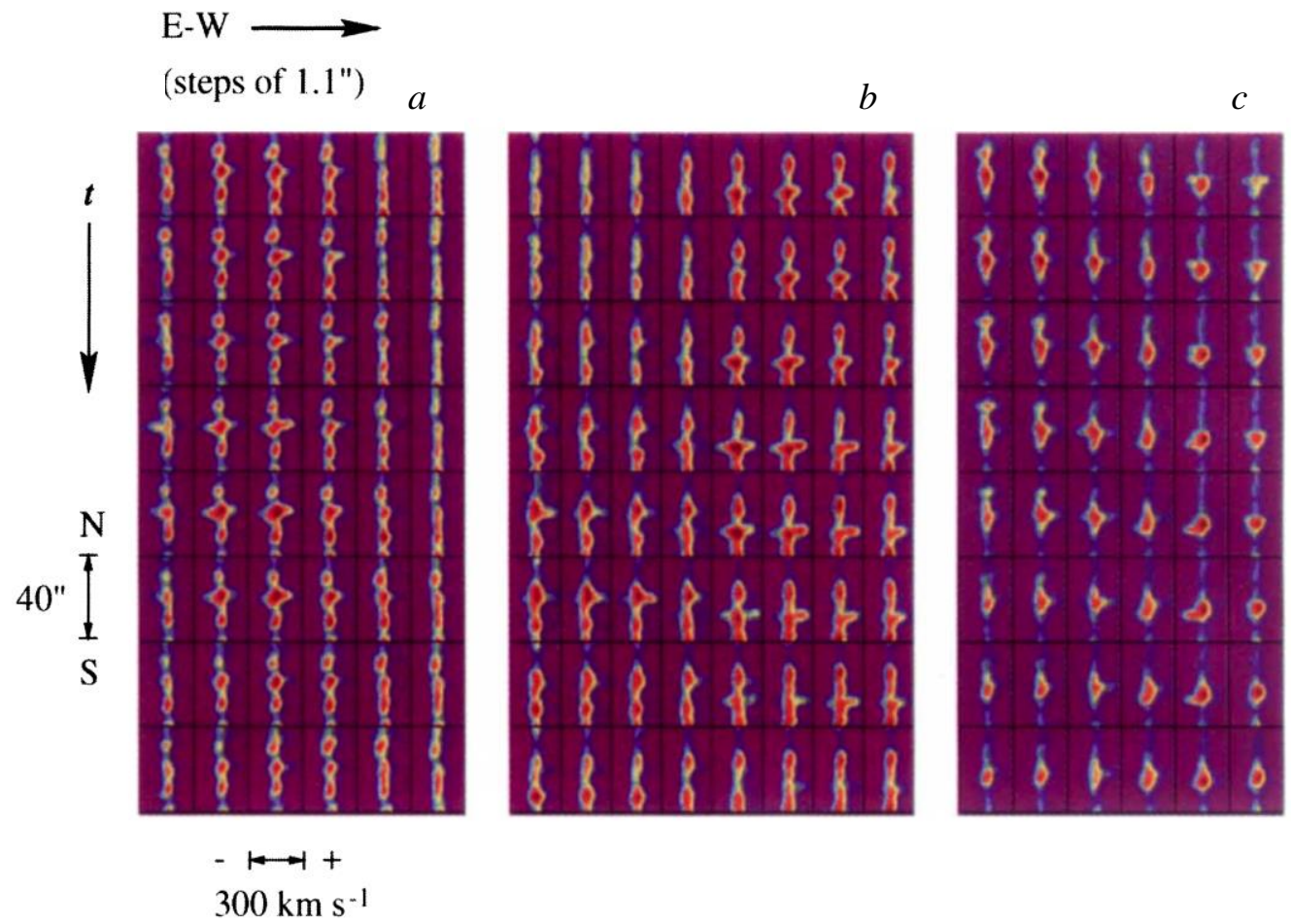

Figure 3. Spectra of TREEs as scanned by SUMER (adapted from [Innes et al., 1997])

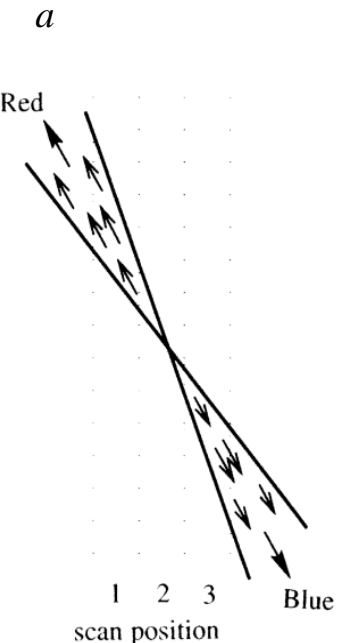

$b$

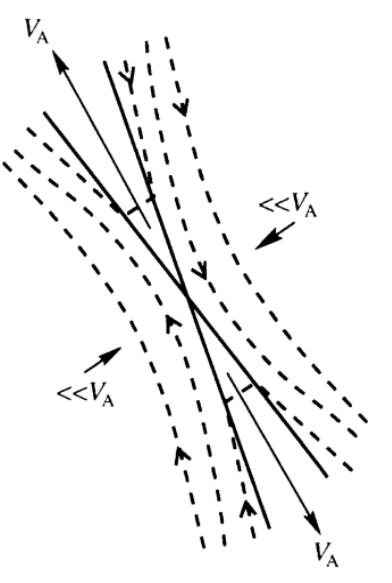

Figure 4. Magnetic reconnection scenario for TREEs based on the observations shown in Figure 3. The spectrograph scanning different locations (1-3) of the reconnection site, the spectra could present blue-wing enhancement, both wing enhancement and red-wing enhancement (adapted from [Innes et al., 1997])

Huang et al. [2018] have found that magnetic braidings of a transition region loop system can create plasma jets at the Alfvén speed with TREE spectra observed in their footpoints. The observations made by Huang et al. [2018] confirm the suggestion that magnetic braiding is also a possible mechanism for producing TREEs [Huang et al., 2017].

Based on these new observations, some modeling attempts have been put forward. Innes et al. [2015] have performed a two-dimensional simulation. They have found that TREE profiles can be reproduced with the multiple magnetic islands and acceleration sites that characterize the plasmoid instability if TREEs are observational signatures of magnetic reconnection sites.

\section{UV BURSTS IN THE TR}

Loop interactions in the stages of flux emergence can emit spectra with characteristics more than those of the TREEs. Peter et al. [2014] have reported on a new type of small-scale events seen in IRIS transition region observations, named "hot explosions" (see, e.g., Figure 6).

In the IRIS imaging data, these events are transient compact bright features with intensity 100-1000 times higher than the median background and a size of a few arcseconds. These events are observed in TR Si IV lines showing very broadened profiles together with clear signatures of chromospheric absorption lines (e.g., Fe II and Ni II blended in the blue wing of Si IV $1394 \AA$; and $\mathrm{Fe}$ II, in the red wing of Si IV $1403 \AA$ ). These events are also called "IRIS bombs" [Vissers et al., 2015; Tian et al., 2016]. Since both IRIS bombs and TREEs have similar size and emit similar broad TR spectra, the blends of cold absorption lines are the main differences that distinguish these events from TREEs. An ISSI group had been assembled aiming at studying these events, and they introduced a term "UV bursts" in the TR to describe small, intense, transient brightenings in ultraviolet images of solar active regions including IRIS bombs as described above (see the definition in [Young et al., 2018]).

Many UV bursts are believed to be the result of magnetic reconnection at the early stage of flux emergence, when the flux tube might show a serpentine (or U-loop) geometry in the lower solar atmosphere [Fan, 2001; Pariat et al., 2004, 2009; Cheung et al., 2007, 2008; Archontis et al., 2009; Cheung, Isobe, 2014; 


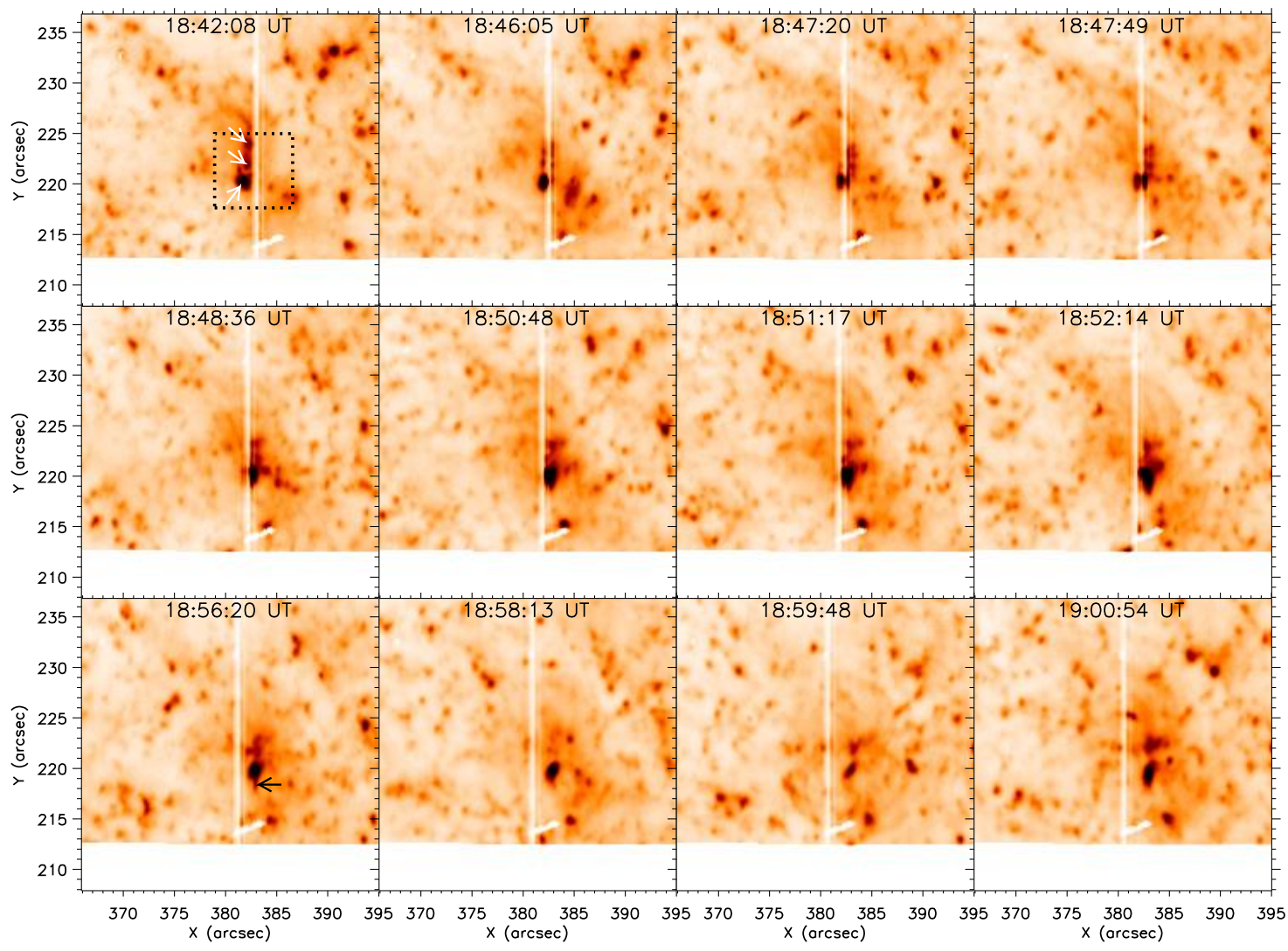

Figure 5. Temporal evolution of a TREE (pointed by arrows in the top-left panel) as seen in IRIS SJ $1330 \AA$ A. The images are shown in reversed color scale (adapted from [Huang et al., 2014a])
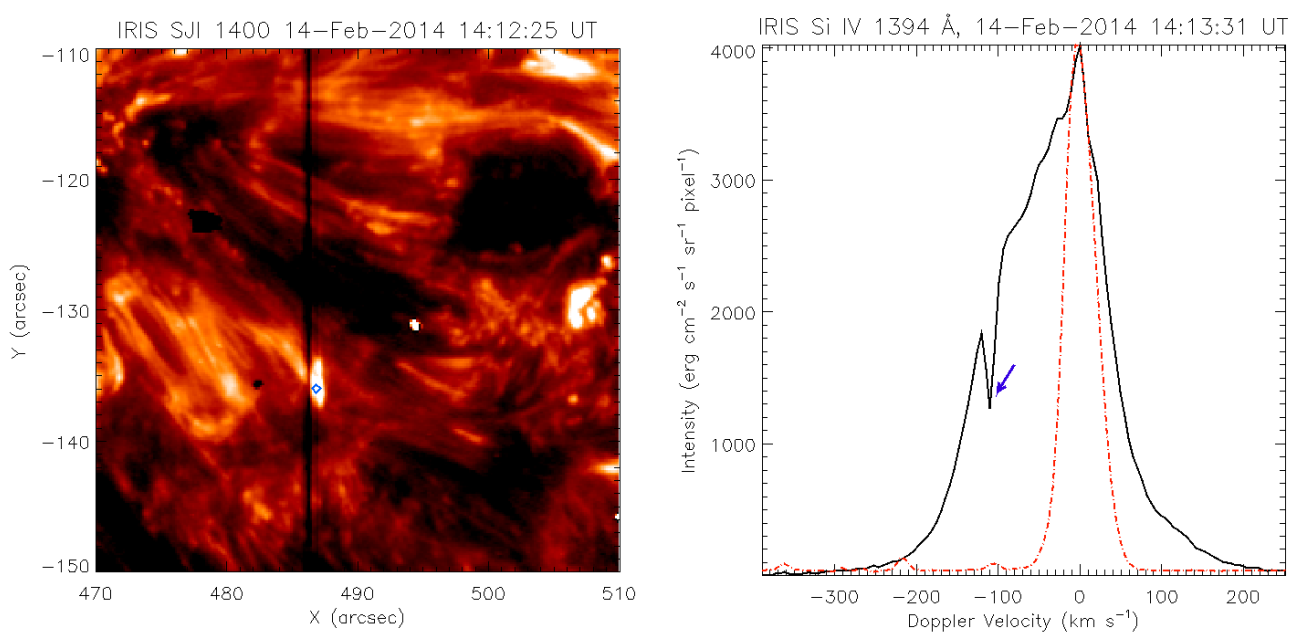

Figure 6. An example of IRIS bomb. The IRIS bomb is the compact bright feature marked by the blue diamond in the IRIS SJ $1400 \AA$ image (left panel). The IRIS Si IV $1394 \AA$ spectrum sampled from the location of the blue diamond is shown on the right panel (black solid line). The blue arrow points at the location where the absorption lines of Ni II are superimposed in the wing of the Si IV line. The red dashed-dotted line on the right panel is the referent spectrum that is taken from a quiet region and multiplied by 9 times. Courtesy of Mr. Zhenyong Hou

Huang et al., 2018]. UV bursts in the TR occur frequently at the early stage of flux emergence (see, e.g. [Peter et al., 2014; Toriumi et al., 2017; Zhao et al., 2017; Tian et al., 2018a] and rarely in the late phase of flux emergence (see, e.g. [Guglielmino et al., 2018; Huang, 2018]). The UV bursts reported in Peter et al. [2014] were first suggested to be caused by magnetic reconnections in the photosphere, which heat plasma to the TR temperature. Judge [2015] performed an independent analysis and suggested that these events were formed in the low to middle chromosphere or above. While Ellerman bombs are phenomena that are well known to be signatures of magnetic reconnections in the photosphere [Ellerman, 1917; Vissers et al., 2015; Reid et al., 2016; Fang et al., 2006; Hong et al., 2014; Nelson et al., 2013, 2015, 2016; Fang et al., 2017; Ni et al., 
2016, 2018a], Tian et al. [2016] have crosschecked IRIS observations and $\mathrm{H} \alpha$ images of 10 TR UV burst events from the Chinese New Vacuum Solar Telescope [Xu et al., 2014; Liu et al., 2014] and have found that 6 of the 10 events are associated with Ellerman bombs in the photosphere and the rest 4 are not. This indicates that some UV bursts in the TR are signatures of magnetic reconnections in the photosphere that heat plasma up to at least the TR temperature [Kim et al., 2015; Tian et al., 2016]. Such a conclusion has also been confirmed by [Nelson et al., 2017], who established that some Ellerman bombs in the quiet Sun also have responses in the IRIS Si IV lines. It is worth mentioning that the blends of the chromospheric absorption lines in the TR emission lines might also be the results of cold plasma (such as filaments, mini-filaments) at the foreground [Vissers et al., 2015; Huang et al., 2017], and thus it is not complete criteria for this species of UV bursts.

TR UV bursts are also diverse. Hou et al. [2016] have reported on a different type of UV bursts, so-called "Narrow-line-width UV bursts" (or "NUBs" for short). NUBs are also compact brightenings but showing very different characteristics in the TR spectra. The TR spectra (Si IV) of NUBs are regularly Gaussian but extremely narrow (less than half of that from a normal region). NUBs are normally found locating above sunspots and might consist of multiple bright cores.

With these observations, some simulation experiments have tried to explain whether magnetic reconnection in the photosphere could heat plasma to transition region temperatures. Ni et al. [2018a, b] have found that initially weakly ionized plasmas can become fully ionized within the reconnection region and the current sheet can be strongly heated to above $2.5 \cdot 10^{4} \mathrm{~K}$ in the cases that the reconnecting magnetic field is in excess of a kilogauss and the plasma $\beta$ is lower than 0.0145 . For more details of UV bursts, we refer to a review paper by Young et al. [2018].

\section{JETS IN THE TR}

Besides the energetic events outstanding from the brightness and spectra, jets also frequently occur in the TR. The IRIS observations have revealed that jets are ubiquitous in the TR network regions and so-called network jets [Tian et al., 2014], and see Figure 7 for examples). Network jets in coronal hole regions are small-scale with widths of $\leq 300 \mathrm{~km}$, lifetimes $20-80 \mathrm{~s}$, and speeds $80-250 \mathrm{~km} / \mathrm{s}$ (see Figure 8 and [Tian et al., 2014; Qi et al., 2019]). Network jets have also been found in the quiet-sun region. These jets are typically slower and shorter than those in coronal holes [Narang et al., 2016; Kayshap et al., 2018]. Some of the network jets should be powered by magnetic reconnection and many could be heated to at least $10^{5} \mathrm{~K}$ [Tian et al., 2014]. The appearances, sizes, lifetimes, and speeds of the network jets have a large overlap with that of spicules (see characteristics of spicules in, e.g., [Xia et al., 2005; De Pontieu et al., 2007; Zhang et al., 2012; Pereira et al., 2012, 2014]. Since both network jets and spicules originate in the network regions, it suggests that at least some of spicules in the chromosphere should be heated to the TR temperature and shown as network jets. A recent simulation by Yang et al. [2018] based on magnetic reconnection driven by a combination of magnetic flux emergence and horizontal advection shows that both fast warm jets (much similar to the network jets) and slow cool jets (much similar to classical spicules) could be generated at a time. Recent analytical studies and simulations on magnetic reconnections driven by magnetic cancellation [Priest et al., 2018; Syntelis et al., 2019] could release energy at different height thus produce energetic events at different temperatures.

Chen et al. [2019] have carried out a statistic analysis aiming at searching for the connection between TREEs and network jets. They have found that (1) TREEs with double peaks or enhancements in both wings appear to be located at either the footpoints of network jets or transient compact brightenings. These TREEs are most likely originated from magnetic reconnection; (2) TREEs with enhancements only at the blue wing are mainly located above network jets along their propagation directions. These TREEs clearly result from the superposition of high-speed network jets onto the TR background; (3) TREEs showing enhancement only at the red wing of the line are generally located around the footpoints of network jets, likely caused by the superposition of reconnection downflows onto the background emission. Their analysis suggests that some TREEs are related to the birth or propagation of network jets, and that other TREEs are not connected to network jets.

Furthermore, Qi et al. [2019] have statistically studied network jets and have found that network jets in the footpoints of strong coronal plumes are more dynamic (higher and faster) than those from regions without clear coronal plumes. This suggests that more dynamic network jets might somehow connect to hotter features in the corona. It has been reported that spicules [De Pontieu et al., 2011; Pant et al., 2015; Jiao et al., 2015, 2016; Samanta et al., 2015] and/or shocks [Hou et al., 2018] could be the possible sources of the propagating

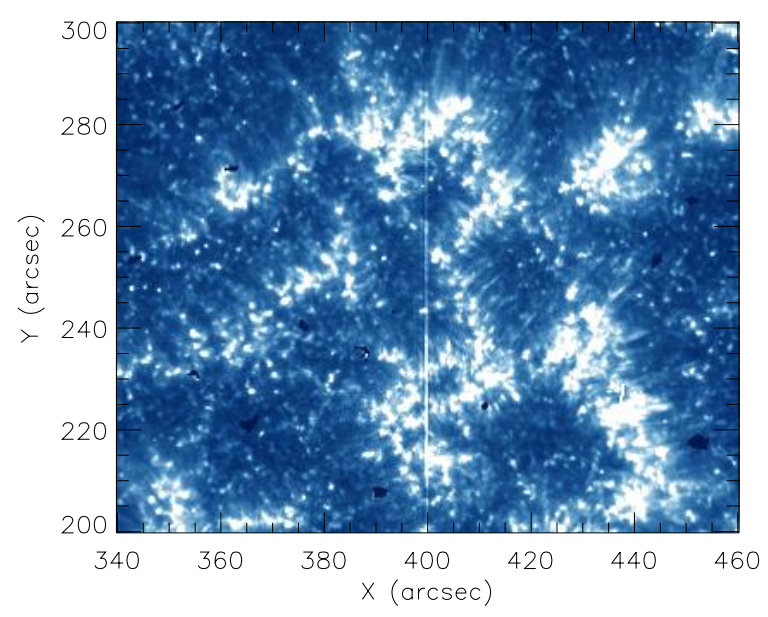

Figure 7. A network region observed in the IRIS SJ $1330 \AA$ passband on December 4, 2015 at 01:29 UT. The compact brightenings are organized to be the so-called "network lanes". The elongated thin features extending from the bright network lanes are network jets. Courtesy of Ms. Youqian Qi 
$a$
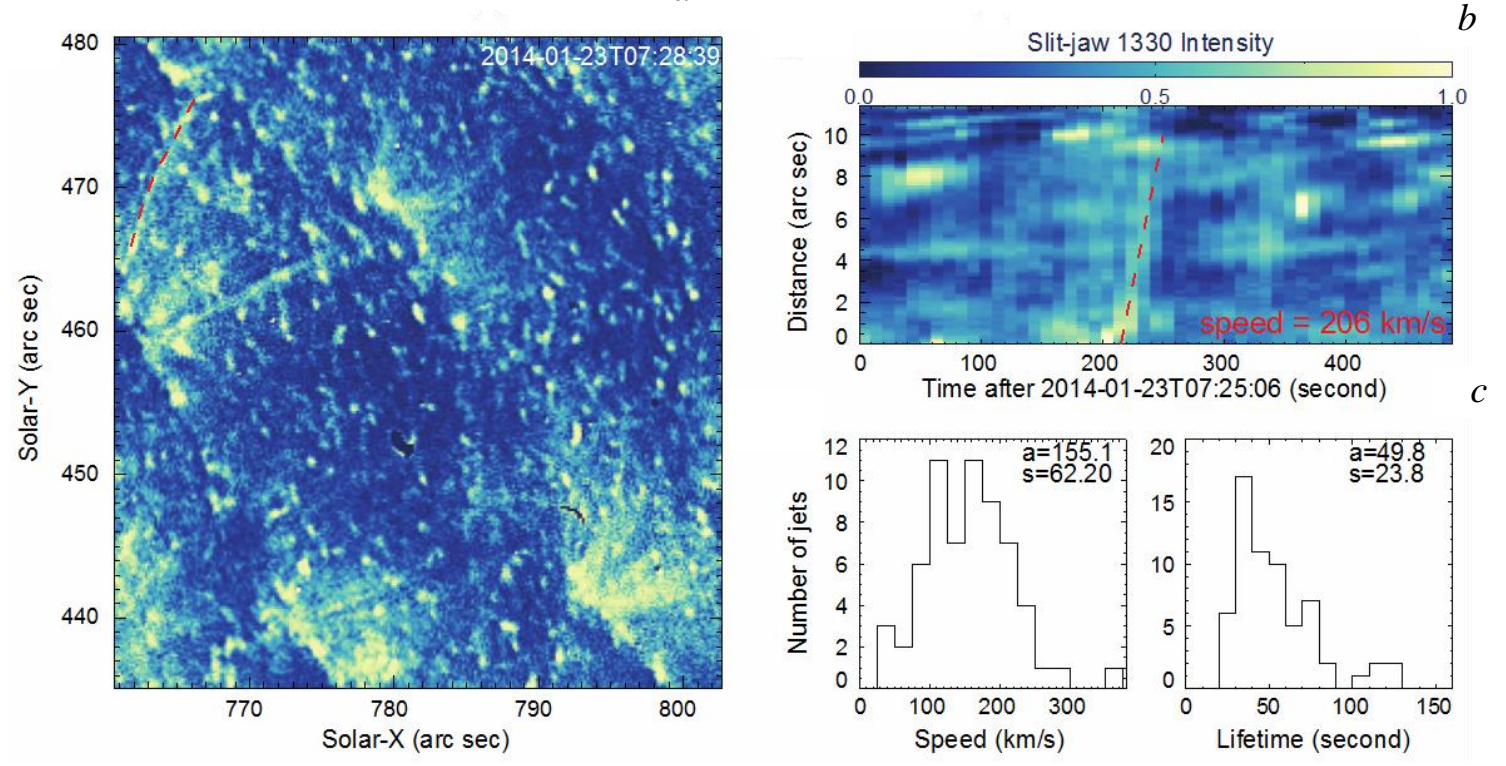

Figure 8. Network and jets seen in IRIS SJ $1330 \AA(a)$, time-distance diagram of a jet $(b)$ and statistic histograms of speeds and lifetimes of network jets (c) (adapted from [Tian et al., 2014])

disturbances in the coronal plumes. It is likely that some of the network jets should also be heated to coronal temperatures.

Besides network jets, there are also some other types of small-scale jets in various regions of the TR. Huang et al. [2018] have reported on very fine jets occurring during the eruption of a braiding structure that contains a quasi-open field. The jets have speeds higher than $300 \mathrm{~km} / \mathrm{s}$, which are comparable to the Alfvén speed in the eruptive region. The observations shown in Huang et al. [2018] also demonstrate that these jets result from magnetic reconnections due to magnetic braiding. Tian et al. [2018b] have reported on high-speed jets found above the light bridge of sunspots. These jets could be clearly seen in images taken at offbands of $\mathrm{H} \alpha$, and they also show signatures of bi-directional flows with speeds as large as $200 \mathrm{~km} / \mathrm{s}$ in TR spectral observations. These jets also exhibit clear inverted Y shapes, which clearly indicate magnetic reconnection between close and open magnetic fields.

\section{CONCLUSION}

The solar transition region (TR) is the crucial interface region between the chromosphere and corona. It is normally defined in the temperature regime from about $2 \cdot 10^{4} \mathrm{~K}$ to about $10^{6} \mathrm{~K}$. The space-born instruments have opened windows for studying this region and many dynamic phenomena have been discovered. This paper reviews a few types of small-scale energetic events occurring in the TR. These events are defined in either spectral or imaging data. Multi-instrument observations available recently have given opportunities to view the same phenomenon in both spectral and imaging data, and it has given more insights to the phenomenon. The observations of these small-scale energetic events indicate the extremely dynamic and complex nature of the TR and they demonstrate that the dynamics of the TR is crucial in the processes of mass and energy transportation between the solar chromosphere and the corona.

The authors would like to thank the referees for their constructive comments and helpful remarks. The authors thank the Organizing Committee of the International Workshop "Eruptive Energy Release Processes on the Sun and Stars: Origins and Effects" for organizing the meeting, especially Dr. Larisa Kashapova for the invitation to write this review. The authors are supported by National Natural Science Foundation of China under contracts of 11761141002, U1831112, 41627806, 41474150 and 41404135. Z.H. thanks the Young Scholar Program of Shandong University, Weihai (2017WHWLJH07). IRIS is a NASA small explorer mission developed and operated by LMSAL with mission operations executed at NASA Ames Research center and major contributions to downlink communications funded by ESA and the Norwegian Space Centre.

\section{REFERENCES}

Alexander C.E., Walsh R.W., Régnier S., Cirtain J., Winebarger A.R., et al. Anti-parallel EUV Flows Observed along Active Region Filament Threads with Hi-C. Astrophys. J. 2013, vol. 775, iss. 1, p. L32. DOI: 10.1088/2041-8205/775/1/L32.

Archontis V., Hood A.W., Savcheva A., Golub L., Deluca E. On the structure and evolution of complexity in sigmoids: a flux emergence model. Astrophys. J. 2009, vol. 691, iss. 2, pp. 12761291. DOI: $10.1088 / 0004-637 X / 691 / 2 / 1276$.

Avrett E.H., Loeser R. Models of the solar chromosphere and transition region from SUMER and HRTS observations: formation of the extreme-ultraviolet spectrum of hydrogen, carbon, and oxygen. Astrophys. J. Supplement Ser. 2008, vol. 175, iss. 1, pp. 229-276. DOI: 10.1086/523671.

Bartoe J.-D.F., Brueckner G.E. New stigmatic, coma-free, concave-grating spectrograph. J. Opt. Soc. Am. 1975, vol. 65, pp. 13-21.

Brueckner G.E., Bartoe J.-D.F. Observations of highenergy jets in the corona above the quiet Sun, the heating of the corona, and the acceleration of the solar wind. Astrophys. $J$. 
1983, vol. 272, pp. 329-348. DOI: 10.1086/161297.

Brueckner G.E., Bartoe J.-D.F., Cook J.W., Dere K.P., Socker D., Kurokawa H., McCabe M. Plasma motions in an emerging flux region. Astrophys. J. 1988, vol. 335, pp. 986995. DOI: $10.1086 / 166985$.

Chae J., Wang H., Lee C.-Y., Goode P.R., Schühle U. Photospheric magnetic field changes associated with transition region explosive events. Astrophys. J. 1998a, vol. 497, iss. 2, pp. L109-L112. DOI: 10.1086/311289.

Chae J., Wang H., Lee C.-Y., Goode P.R., Schühle U. Chromospheric upflow events associated with transition region explosive events. Astrophys. J. 1998b, vol. 504, iss. 2, pp. L123-L126. DOI: 10.1086/311583.

Chen Y.J., Tian H., Huang Z., Peter H., Samanta T. Investigating the transition region explosive events and their relationship to network jets. Astrophys. J. 2019, vol. 873, iss. 1, article id. 79,9 p. DOI: $10.3847 / 1538-4357 / a b 0417$.

Cheung M.C.M., Schüssler M., Moreno-Insertis F. Magnetic flux emergence in granular convection: radiative MHD simulations and observational signatures. Astron. Astrophys. 2007 vol. 467, iss. 2, pp. 703-719. DOI: 10.1051/0004-6361:20077048.

Cheung M.C.M., Schüssler M., Tarbell T.D., Title A.M. Solar surface emerging flux regions: a comparative study of radiative MHD modeling and Hinode SOT observations. Astrophys. J. 2008, vol. 687, iss. 2, pp. 1373-1387. DOI: 10.1086/591245.

Cheung M.C.M., Isobe H. Flux emergence (Theory). Living Reviews in Solar Phys. 2014, vol. 11, iss. 1, article id. 3, 128 p. DOI: 10.12942/lrsp-2014-3.

Cirtain J.W., Golub L., Winebarger A.R., De Pontieu B., Kobayashi K., Moore R.L., et al. Energy release in the solar corona from spatially resolved magnetic braids. Nature. 2013, vol. 493, iss. 7433, pp. 501-503. DOI: 10.1038/nature11772.

Culhane J.L., Harra L.K., James A.M., Al-Janabi K., Bradley L.J., Chaudry R.A., et al. The EUV Imaging Spectrometer for Hinode. Solar Phys. 2007, vol. 243, iss. 1, pp. 1961. DOI: 10.1007/s01007-007-0293-1.

Curdt W., Tian H. Spectroscopic evidence for helicity in explosive events. Astron. Astrophys. 2011, vol. 532, no. L9, 4 p. DOI: 10.1051/0004-6361/201117116.

De Pontieu B., McIntosh S., Hansteen V.H., Carlsson M., Schrijver C.J., Tarbell T.D., et al. A tale of two spicules: the impact of spicules on the magnetic chromosphere. Publ. of the Astron. Soc. of Japan. 2007, vol. 59, no. SP3, pp. S655-S662. DOI: 10.1093/pasj/59.sp3.S655.

De Pontieu B., McIntosh S.W., Carlsson M., Hansteen V.H., Tarbell T.D., Boerner P., et al. The origins of hot plasma in the solar corona. Science. 2011, vol. 331, iss. 6013, pp. 55-58. DOI: $10.1126 /$ science.1197738.

De Pontieu B., Rouppe van der Voort L., McIntosh S.W., Pereira T.M.D., Carlsson M., et al. On the prevalence of small-scale twist in the solar chromosphere and transition region. Science. 2014a, vol. 346, iss. 6207, id. 1255732. DOI: $10.1126 /$ science.1255732.

De Pontieu B., Title A.M., Lemen J.R., Kushner G.D., Akin D.J., Allard B., et al. The Interface Region Imaging Spectrograph (IRIS). Solar Phys. 2014b, vol. 289, iss. 7, pp. 2733-2779. DOI: 10.1007/s11207-014-0485-y.

Dere K.P. Explosive events and magnetic reconnection in the solar atmosphere. Solar Wind Seven Colloquium. Eds. E. Marsch, R. Schwenn. Oxford, Pergamon Press, 1992, pp. 11-20.

Dere K.P., Bartoe J.-D.F., Brueckner G.E. High-resolution telescope and spectrograph observations of the quiet solar chromosphere and transition zone. Astrophys. J. 1984, vol. 281, pp. $870-883$. DOI: $10.1086 / 162167$.

Dere K.P., Bartoe J.-D.F., Brueckner G.E. Explosive events in the solar transition zone. Solar Phys. 1989, vol. 123, pp. 41-68. DOI: 10.1007/BF00150011.

Dere K.P., Bartoe J.-D.F., Brueckner G.E., Ewing J., Lund P.
Explosive events and magnetic reconnection in the solar atmosphere. J. Geophys. Res. 1991, vol. 96, pp. 9399-9407. DOI: 10.1029/90JA02572.

Domingo V., Fleck B., Poland A.I. The SOHO mission: an overview. Solar Phys. 1995, vol. 162, iss. 1-2, pp. 1-37. DOI: 10.1007/BF00733425.

Ellerman F. Solar hydrogen "bombs". Astrophys. J. 1917, vol. 46, p. 298. DOI: 10.1086/142366.

Fan Y. The emergence of a twisted $\Omega$-tube into the solar atmosphere. Astrophys. J. 2001, vol. 554, iss. 1, pp. L111-L114. DOI: $10.1086 / 320935$

Fang C., Tang Y.H., Xu Z., Ding M.D., Chen P.F. Spectral analysis of Ellerman bombs. Astrophys. J. 2006, vol. 643, iss. 2, pp. 1325-1336. DOI: $10.1086 / 501342$.

Fang C., Hao Q., Ding M.-D., Li Z. Can the temperature of Ellerman bombs be more than $10000 \mathrm{~K}$ ? Res. Astron. Astrophys. 2017, vol. 17, iss. 4, article id. 031. DOI: 10.1088/16744527/ $17 / 4 / 31$.

Golub L., Deluca E., Austin G., Bookbinder J., Caldwell D., Cheimets P., et al. The X-Ray Telescope (XRT) for the Hinode mission. Solar Phys. 2007, vol. 243, iss. 1, pp. 63-86. DOI: $10.1007 / \mathrm{s} 11207-007-0182-1$.

Guglielmino S.L., Zuccarello F., Young P.R., Murabito M., Romano P. IRIS observations of magnetic interactions in the solar atmosphere between preexisting and emerging magnetic fields. I. Overall evolution. Astrophys. J. 2018, vol. 856, iss. 2, article id. 127, 15 p. DOI: 10.3847/1538-4357/aab2a8.

Handy B.N., Acton L.W., Kankelborg C.C., Wolfson C.J., Akin D.J., Bruner M.E., et al. The transition region and coronal explorer. Solar Phys. 1999, vol. 187, iss. 2, pp. 229-260. DOI: 10.1023/A:1005166902804.

Hansteen V., De Pontieu B., Carlsson M., Lemen J., Title A., Boerner P., et al. The unresolved fine structure resolved: IRIS observations of the solar transition region. Science. 2014, vol. 346, iss. 6207 , id. 1255757 . DOI: $10.1126 /$ science. 1255757.

Harrison R.A. EUV blinkers: the significance of variations in the extreme ultraviolet quiet Sun. Solar Phys. 1997, vol. 175, iss. 2, pp. 467-485. DOI: 10.1023/A:1004964707047.

Harrison R.A., Sawyer E.C., Carter M.K., Cruise A.M., Cutler R.M., Fludra A., et al. The Coronal Diagnostic Spectrometer for the Solar and Heliospheric Observatory. Solar Phys. 1995, vol. 162, iss. 1-2, pp. 233-290. DOI: 10.1007/BF00733431.

Hassler D.M., Dammasch I.E., Lemaire P., Brekke P., Curdt W., Mason H.E., et al. Solar wind outflow and the chromospheric magnetic network. Science. 1999, vol. 283, iss. 5403, p. 810. DOI: $10.1126 /$ science.283.5403.810.

Hong J., Ding M.D., Li Y., Fang C., Cao W. Spectral observations of Ellerman bombs and fitting with a two-cloud model. Astrophys. J. 2014, vol. 792, iss. 1, article id. 13, 10 p. DOI: 10.1088/0004-637X/792/1/13.

Hou Z., Huang Z., Xia L., Li B., Madjarska M.S., Fu H., et al. Narrow-line-width UV bursts in the transition region above sunspots observed by IRIS. Astrophys. J. 2016, vol. 829, iss. 2, article id. L30, 7 p. DOI: 10.3847/2041-8205/829/2/L30.

Hou Z., Huang Z., Xia L., Li B., Fu H. Observations of upward propagating waves in the transition region and corona above sunspots. Astrophys. J. 2018, vol. 855, iss. 1, article id. 65, 12 p. DOI: $10.3847 / 1538-4357 / a a a b 5 a$.

Huang Z. Magnetic loops above a small flux-emerging region observed by IRIS, Hinode, and SDO. Astrophys. J., 2018, vol. 869 , iss. 2 , article id. 175,13 p. DOI: $10.3847 / 1538-$ 4357/aaef86.

Huang Z., Madjarska M.S., Doyle J.G., Lamb D.A. Coronal hole boundaries at small scales. IV. SOT view. Magnetic field properties of small-scale transient brightenings in coronal holes. Astron. Astrophys. 2012, vol. 548, id. A62, 19 p. DOI: 10.1051/0004-6361/201220079.

Huang Z., Madjarska M.S., Xia L., Doyle J.G., Galsgaard K., 
$\mathrm{Fu} \mathrm{H}$. Explosive events on a subarcsecond scale in IRIS observations: a case study. Astrophys. J. 2014a, vol. 797, iss. 2, article id. 88, 14 p. DOI: 10.1088/0004-637X/797/2/88.

Huang Z., Madjarska M.S., Koleva K., Doyle J.G., Duchlev P., Dechev M., Reardon K. H $\alpha$ spectroscopy and multiwavelength imaging of a solar flare caused by filament eruption. Astron. Astrophys. 2014b, vol. 566, id. A148, 18 p. DOI: 10.1051/0004-6361/201323097.

Huang Z., Xia L., Li B., Madjarska M.S. Cool transition region loops observed by the Interface Region Imaging Spectrograph. Astrophys. J. 2015, vol. 810, iss. 1, article id. 46, 11 p DOI: 10.1088/0004-637X/810/1/46.

Huang Z., Madjarska M.S., Scullion E.M., Xia L.-D., Doyle J.G., Ray T. Explosive events in active region observed by IRIS and SST/CRISP. Monthly Notices of the Royal Astron. Soc. 2017, vol. 464, iss. 2, pp. 1753-1761. DOI: 10.1093/ mnras/stw2469.

Huang Z., Mou C., Fu H., Deng L., Li B., Xia L. A magnetic reconnection event in the solar atmosphere driven by relaxation of a twisted arch filament system. Astrophys. $J$. 2018 , vol. 853 , iss. 2 , article id. L26, 8 p. DOI: $10.3847 / 2041$ 8213/aaa88c.

Innes D.E., Inhester B., Axford W.I., Wilhelm K. Bidirectional plasma jets produced by magnetic reconnection on the Sun. Nature, 1997, vol. 386, iss. 6627, pp. 811-813. DOI: $10.1038 / 386811 \mathrm{a} 0$.

Innes D.E., Guo L.-J., Huang Y.-M., Bhattacharjee A. IRIS Si IV line profiles: an indication for the plasmoid instability during small-scale magnetic reconnection on the Sun. Astrophys. J. 2015, vol. 813, iss. 2, article id. 86, 11 p. DOI: $10.1088 / 0004-637 \mathrm{X} / 813 / 2 / 86$.

Jiao F., Xia L., Li B., Huang Z., Li X., Chandrashekhar K., et al. Sources of quasi-periodic propagating disturbances above a solar polar coronal hole. Astrophys. J. 2015, vol. 809, iss. 1, article id. L17, 5 p. DOI: 10.1088/2041-8205/809/1/L17.

Jiao F.-R., Xia L.-D., Huang Z.-H., Li B., Fu H., Yuan D., Chandrashekhar K. Damping and power spectra of quasi-periodic intensity disturbances above a solar polar coronal hole. Res. Astron. Astrophys. 2016, vol. 16, iss. 6, article id. 93. DOI: 10.1088/ 1674-4527/16/6/093.

Judge P.G. UV spectra, bombs, and the solar atmosphere. Astrophys. J. 2015, vol. 808, iss. 2, article id. 116, 5 p. DOI: $10.1088 / 0004-637 X / 808 / 2 / 116$.

Kayshap P., Murawski K., Srivastava A.K., Dwivedi B.N. Rotating network jets in the quiet Sun as observed by IRIS. Astron. Astrophys. 2018, vol. 616, article id. A99, 8 p. DOI: 10.1051/0004-6361/201730990.

Kim Y.-H., Yurchyshyn V., Bong S.-C. Simultaneous observation of a hot explosion by NST and IRIS. Astrophys. $J$. 2015, vol. 810, iss. 1, article id. 38, 12 p. DOI: 10.1088/0004$637 X / 810 / 1 / 38$

Klimchuk J.A. On solving the coronal heating problem. Solar Phys. 2006, vol. 234, iss. 1, pp. 41-77. DOI: 10.1007/s11207006-0055-z.

Kosugi T., Matsuzaki K., Sakao T., Shimizu T., Sone Y., Tachikawa S., et al. The Hinode (Solar-B) mission: an overview. Solar Phys. 2007, vol. 243, iss. 1, pp. 3-17. DOI: 10.1007/ s11207-007-9014-6.

Lemaire P., Wilhelm K., Curdt W., Schule U., Marsch E., Poland A.I., et al. First results of the SUMER telescope and spectrometer on SOHO. II. Imagery and data management. Solar Phys. 1997, vol. 170, iss. 1, pp. 105-122. DOI: 10.1023/ A: 1004994702333 .

Lemen J.R., Title A.M., Akin D.J., Boerner P.F., Chou C., Drake J.F., et al. The Atmospheric Imaging Assembly (AIA) on the Solar Dynamics Observatory (SDO). Solar Phys. 2012, vol. 275, iss. 1-2, pp. 17-40. DOI: 10.1007/s11207-011-9776-8.

Li D. Transition-region explosive events produced by plas- moid instability. Res. Astron. Astrophys. 2019, vol. 19, no. 5, article id. 67, 10 p. DOI: 10.1088/1674-4527/19/5/67.

Li L., Zhang J., Peter H., Priest E., Chen H., Guo L., et al. Magnetic reconnection between a solar filament and nearby coronal loops. Nature Phys. 2016, vol. 12, iss. 9, pp. 847-851. DOI: $10.1038 /$ nphys3768.

Li L., Zhang J., Peter H., Chitta L.P., Su J., Xia C., et al. Coronal condensations caused by magnetic reconnection between solar coronal loops. Astrophys. J. 2018a, vol. 864, iss. 1, article id. L4, 7 p. DOI: 10.3847/2041-8213/aad90a.

Li D., Li L., Ning Z. Spectroscopic and imaging observations of small-scale reconnection events. Monthly Notices Royal Astron. Soc. 2018b, vol. 479, iss. 2, pp. 2382-2388. DOI: $10.1093 / \mathrm{mnras} /$ sty1712.

Liu Z., Xu J., Gu B.-Z., Wang S., You J.-Q., Shen L.-X., et al. New vacuum solar telescope and observations with high resolution. Res. Astron. Astrophys. 2014, vol. 14, iss. 6, pp. 705-718. DOI: 10.1088/1674-4527/14/6/009.

Madjarska M.S., Doyle J.G. Temporal evolution of different temperature plasma during explosive events. Astron. Astrophys. 2002, vol. 382, pp. 319-327. DOI: 10.1051/0004-6361:20011610.

Madjarska M.S., Doyle J.G. Simultaneous observations of solar transition region blinkers and explosive events by SUMER, CDS and BBSO. Are blinkers, explosive events and spicules the same phenomenon? Astron. Astrophys. 2003, vol. 403, pp. 731741. DOI: 10.1051/0004-6361:20030397.

Madjarska M.S., Doyle J.G., De Pontieu B. Explosive events associated with a surge. Astrophys. J. 2009, vol. 701, iss. 1, pp. 253-259. DOI: 10.1088/0004-637X/701/1/253.

Madjarska M.S., Huang Z., Doyle J.G., Subramanian S. Coronal hole boundaries evolution at small scales. III. EIS and SUMER views. Astron. Astrophys. 2012, vol. 545, article id. A67, 16 p. DOI: 10.1051/0004-6361/201219516.

Mariska J.T. The quiet solar transition region. Ann. Rev. Astron. Astrophys. 1986, vol. 24. pp. 23-48. DOI: 10.1146/ annurev.aa.24.090186.000323.

Mariska J.T. The Solar Transition Region. New York, Cambridge University Press, 1992, 290 p.

Mou C., Huang Z., Xia L., Madjarska M.S., Li B., Fu H., Jiao F., Hou Z. Magnetic flux supplement to coronal bright points. Astrophys. J. 2016, vol. 818, iss. 1, article id. 9, 13 p. DOI: 10.3847/0004-637X/818/1/9.

Muglach K. Explosive events and the evolution of the photospheric magnetic field. Astrophys. J. 2008, vol. 687, iss. 2, pp. 1398-1405. DOI: 10.1086/592065.

Narang N., Arbacher R.T., Tian H., Banerjee D., Cranmer S.R., DeLuca E.E., McKillop S. Statistical study of network jets observed in the solar transition region: a comparison between coronal holes and quiet-Sun regions. Solar Phys. 2016, vol. 291, iss. 4, pp. 1129-1142. DOI: 10.1007/s11207-016-0886-1.

Nelson C.J., Shelyag S., Mathioudakis M., Doyle J.G., Madjarska M.S., Uitenbroek H., Erdélyi R. Ellerman bombsevidence for magnetic reconnection in the lower solar atmosphere. Astrophys. J. 2013, vol. 779, iss. 2, article id. 125, 10 p. DOI: 10.1088/0004-637X/779/2/125.

Nelson C.J., Scullion E.M., Doyle J.G., Freij N., Erdélyi R. Small-scale structuring of Ellerman bombs at the solar limb. Astrophys. J. 2015, vol. 798, iss. 1, article id. 19, 9 p. DOI: 10.1088/0004-637X/798/1/19.

Nelson C.J., Doyle J.G., Erdélyi R. Statistical study of network jets observed in the solar transition region: a comparison between coronal holes and quiet-Sun regions. Monthly Notices Royal Astron. Soc. 2016, vol. 463, iss. 2, pp. 2190 2201. DOI: 10.1093/mnras/stw2034.

Nelson C.J., Freij N., Reid A., Oliver R., Mathioudakis M., Erdélyi R. IRIS burst spectra co-spatial to a quiet-Sun Ellermanlike brightening. Astrophys. J. 2017, vol. 845, iss. 2, article id. 16, 11 p. DOI: $10.3847 / 1538-4357 / a a 7 e 7 a$. 
Ni L., Lin J., Roussev I.I., Schmieder B. Heating mechanisms in the low solar atmosphere through magnetic reconnection in current sheets. Astrophys. J. 2016, vol. 832, iss. 2, article id. 195, 11 p. DOI: 10.3847/0004-637X/832/2/195.

Ni L., Lukin V.S., Murphy N.A., Lin J. Magnetic reconnection in strongly magnetized regions of the low solar chromosphere. Astrophys. J. 2018a, vol. 852, iss. 2, article id. 95, 11 p. DOI: 10.3847/1538-4357/aa9edb.

Ni L., Lukin V.S., Murphy N.A., Lin J. Magnetic reconnection in the low solar chromosphere with a more realistic radiative cooling model. Phys. Plasmas. 2018b, vol. 25, iss. 4, id. 042903. DOI: $10.1063 / 1.5018351$

Ning Z., Innes D., Solanki S. Line profile characteristics of solar explosive event bursts. Astron. Astrophys. 2004, vol. 419, pp. 1141-1148. DOI: 10.1051/0004-6361:20034499.

Pant V., Dolla L., Mazumder R., Banerjee D., Krishna Prasad S., Panditi V. Dynamics of on-disk plumes as observed with the Interface Region Imaging Spectrograph, the Atmospheric Imaging Assembly, and the Helioseismic and Magnetic Imager. Astrophys. J. 2015, vol. 807, iss. 1, article id. 71, 12 p. DOI: $10.1088 / 0004-637 X / 807 / 1 / 71$.

Pariat E., Aulanier G., Schmieder B., Georgoulis M.K., Rust D.M., Bernasconi P.N. Resistive emergence of undulatory flux tubes. Astrophys. J. 2004, vol. 614, iss. 2, pp. 1099-1112 DOI: $10.1086 / 423891$.

Pariat E., Masson S., Aulanier G. Current buildup in emerging serpentine flux tubes. Astrophys. J. 2009, vol. 701, iss. 2, pp. 1911-1921. DOI: 10.1088/0004-637X/701/2/1911.

Parker E.N. Magnetic neutral sheets in evolving fields. I. General theory. Astrophys. J. 1983a, vol. 264, p. 635 DOI: 10.1086/160636.

Parker E.N. Magnetic neutral sheets in evolving fields. II Formation of the solar corona. Astrophys. J. 1983b, vol. 264 p. 642 . DOI: $10.1086 / 160637$.

Parker E.N. Nanoflares and the solar X-ray corona. Astrophys. J. 1988, vol. 330, pp. 474-479. DOI: 10.1086/166485.

Pereira T.M.D., De Pontieu B., Carlsson M. Quantifying spicules. Astrophys. J. 2012, vol. 759, iss. 1, article id. 18, 16 p. DOI: 10.1088/0004-637X/759/1/18.

Pereira T.M.D., De Pontieu B., Carlsson M., Hansteen V., Tarbell T.D., Lemen J., Title A., Boerner P., Hurlburt N., et al. An Interface Region Imaging Spectrograph first view on solar spicules. Astrophys. J. 2014, vol. 792, iss. 1, article id. L15, 6 p. DOI: 10.1088/2041-8205/792/1/L15.

Pesnell W.D., Thompson B.J., Chamberlin P.C. The Solar Dynamics Observatory (SDO). Solar Phys. 2012, vol. 275, iss. 1-2, pp. 3-15. DOI: 10.1007/s11207-011-9841-3.

Peter H., Tian H., Curdt W., Schmit D., Innes D., De Pontieu B., Lemen J., Title A., Boerner P., Hurlburt N., et al. Hot explosions in the cool atmosphere of the Sun. Science. 2014, vol. 346, iss. 6207, article id. 1255726. DOI: 10.1126 science. 1255726.

Porter J.G., Dere K.P. The magnetic network location of explosive events observed in the solar transition region. Astrophys. J. 1991, vol. 370, pp. 775-778. DOI: 10.1086/169860.

Priest E. Magnetohydrodynamics of the Sun. Cambridge, Cambridge University Press, 2014, 582 p. DOI: 10.1017/CBO 9781139020732.

Priest E., Forbes T. Magnetic Reconnection: MHD Theory and Applications. New York, Cambridge University Press, 2000, 597 p. DOI: 10.1017/CBO9780511525087.

Priest E.R., Chitta L.P., Syntelis P. A cancellation nanoflare Model for solar chromospheric and coronal heating. Astrophys. J. Lett. 2018, vol. 862, iss. 2, article id. L24, 7 p. DOI: $10.3847 / 2041-8213 /$ aad4fc.

Qi Y.Q., Huang Z., Xia L., Li B., Fu H. Liu W., Sun M., Hou Z. On the relation between transition region network jets and coronal plumes. Solar Phys. 2019. [Under review].
Reid A., Mathioudakis M., Doyle J.G., Scullion E., Nelson C.J., Henriques V., Ray T. Magnetic flux cancellation in Ellerman bombs. Astrophys. J. 2016, vol. 823, iss. 2, article id. 110, 10 p. DOI: $10.3847 / 0004-637 \mathrm{X} / 823 / 2 / 110$.

Samanta T., Pant V., Banerjee D. Propagating disturbances in the solar corona and spicular connection. Astrophys. $J$. 2015, vol. 815, iss. 1, article id. L16, 6 p. DOI: 10.1088/20418205/815/1/L16.

Scherrer P.H., Schou J., Bush R.I., Kosovichev A.G., Bogart R.S., Hoeksema J.T., Liu Y., Duvall T.L., Zhao J., Title A.M., Schrijver C.J., Tarbell T.D., Tomczyk S. The Helioseismic and Magnetic Imager (HMI) investigation for the Solar Dynamics Observatory (SDO). Solar Phys. 2012, vol. 275, iss. 1-2, pp. 207227. DOI: 10.1007/s11207-011-9834-2.

Schrijver C.J. Braiding-induced interchange reconnection of the magnetic field and the width of solar coronal loops. Astrophys. J. 2007, vol. 662, iss. 2, pp. L119-L122. DOI: 10.1086/519455.

Su Y., Veronig A.M., Holman G.D., Dennis B.R., Wang T., Temmer M., Gan W. Imaging coronal magnetic-field reconnection in a solar flare. Nature Phys. 2013, vol. 9, iss. 8, pp. 489-493. DOI: 10.1038/nphys2675.

Sun J.Q., Cheng X., Ding M.D., Guo Y., Priest E.R., Parnell C.E., Edwards S.J., Zhang J., Chen P.F., Fang C. Extreme ultraviolet imaging of three-dimensional magnetic reconnection in a solar eruption. Nature Communications. 2015, vol. 6, article id. 7598, 7 p. DOI: 10.1038/ncomms8598.

Syntelis P., Priest E.R., Chitta L.P. A cancellation nanoflare model for solar chromospheric and coronal heating. II. 2D theory and simulations. Astrophys. J. 2019, vol. 872, iss. 1, article id. 32, 15 p. DOI: $10.3847 / 1538-4357 /$ aafaf8.

Teriaca L., Banerjee D., Falchi A., Doyle J.G., Madjarska M.S. Transition region small-scale dynamics as seen by SUMER on SOHO. Astron. Astrophys. 2004, vol. 427, pp. 10651074. DOI: 10.1051/0004-6361:20040503.

Testa P., De Pontieu B., Allred J., Carlsson M., Reale F., Daw A., Hansteen V., Martinez-Sykora J., Liu W., deLuca E.E., Golub L., et al. Evidence of nonthermal particles in coronal loops heated impulsively by nanoflares. Science. 2014, vol. 346, iss. 6207, id. 1255724. DOI: 10.1126/science. 1255724 .

Tian H. Probing the solar transition region: current status and future perspectives. Res. Astron. Astrophys. 2017, vol. 17, iss. 11, article id. 110, 18 p. DOI: 10.1088/1674-4527/17/11/110.

Tian H., DeLuca E.E., Cranmer S.R., De Pontieu B., Peter H., Martínez-Sykora J., Golub L., McKillop S., Reeves K.K., et al. Prevalence of small-scale jets from the networks of the solar transition region and chromosphere. Science. 2014, vol. 346, iss. 6207, id. 1255711. DOI: 10.1126/science.1255711.

Tian H., Xu Z., He J., Madsen C. Are IRIS bombs connected to Ellerman bombs? Astrophys. J. 2016, vol. 824, iss. 2, article id. 96, 14 p. DOI: 10.3847/0004-637X/824/2/96.

Tian H., Zhu X., Peter H., Zhao J., Samanta T., Chen Y. Magnetic reconnection at the earliest stage of solar flux emergence. Astrophys. J. 2018a, vol. 854, iss. 2, article id. 174, 13 p. DOI: 10.3847/1538-4357/aaaae6.

Tian H., Yurchyshyn V., Peter H., Solanki S.K., Young P.R., Ni L., Cao W., Ji K., Zhu Y., Zhang J., Samanta T., et al. Frequently occurring reconnection jets from sunspot light bridges. Astrophys. J. 2018b vol. 854, iss. 2, article id. 92, 13 p. DOI: $10.3847 / 1538-4357 / a a a 89 d$.

Toriumi S., Katsukawa Y., Cheung M.C.M. Various local heating events in the earliest phase of flux emergence. Astrophys. J. 2017, vol. 836, iss. 1 , article id. 63, 13 p. DOI: $10.3847 / 1538$ 4357/836/1/63

Tsuneta S., Ichimoto K., Katsukawa Y., Nagata S., Otsubo M., Shimizu T., Suematsu Y., Nakagiri M., Noguchi M., Tarbell T., Title A., et al. The Solar Optical Telescope for the Hinode mission: an overview. Solar Phys. 2008, vol. 249, iss. 2, pp. 167-196. DOI: 10.1007/s11207-008-9174-z. 
Tu C.-Y., Zhou C., Marsch E., Xia L.-D., Zhao L., Wang J.-X., Wilhelm K. Solar wind origin in coronal funnels. Science. 2005, vol. 308, iss. 5721, pp. 519-523. DOI: 10.1126/science.1109447.

van Ballegooijen A.A., Martens P.C.H. Formation and eruption of solar prominences. Astrophys. J. 1989, vol. 343, pp. 971-984. DOI: 10.1086/167766.

Vernazza J.E., Avrett E.H., Loeser R. Structure of the solar chromosphere. III. Models of the EUV brightness components of the quiet-Sun. Astrophys. J. Suppl. Ser. 1981, vol. 45 , pp. 635-725. DOI: 10.1086/190731.

Vissers G.J.M., Rouppe van der Voort L.H.M., Rutten R.J., Carlsson M., De Pontieu B. Ellerman bombs at high resolution. III. Simultaneous observations with IRIS and SST. Astrophys. J. 2015 , vol. 812 , iss. 1 , article id. 11,18 p. DOI: $10.1088 / 0004-$ $637 \mathrm{X} / 812 / 1 / 11$

Wedemeyer-Böhm S., Lagg A., Nordlund A. Coupling from the photosphere to the chromosphere and the corona. Space Sci. Rev. 2009, vol. 144, iss. 1-4, pp. 317-350. DOI: 10.1007/ s11214-008-9447-8.

Wilhelm K. Solar spicules and macrospicules observed by SUMER. Astron. Astrophys. 2000, vol. 360, pp. 351-362.

Wilhelm K., Curdt W., Marsch E., Schühle U., Lemaire P., Gabriel A., Vial J.-C., Grewing M., Huber M.C.E., Jordan S.D., Poland A.I., et al. SUMER - Solar Ultraviolet Measurements of Emitted Radiation. Solar Phys. 1995, vol. 162, iss. 1-2, pp. 189-231. DOI: 10.1007/BF00733430.

Xia L.D., Marsch E., Curdt W. On the outflow in an equatorial coronal hole. Astron. Astrophys. 2003, vol. 399, pp. L5-L9. DOI: 10.1051/0004-6361:20030016.

Xia L.D., Marsch E., Wilhelm K. On the network structures in solar equatorial coronal holes. Observations of SUMER and MDI on SOHO. Astron. Astrophys. 2004, vol. 424, pp. 1025-1037. DOI: 10.1051/0004-6361:20047027.

Xia L.D., Popescu M.D., Doyle J.G., Giannikakis J. Time series study of EUV spicules observed by SUMER/SOHO. Astron. Astrophys. 2005, vol. 438, iss. 3, pp. 1115-1122. DOI: 10.1051/0004-6361:20042579.

Xu Z., Jin Z.Y., Xu F.Y., Liu Z. Primary observations of solar filaments using the multi-channel imaging system of the New Vacuum Solar Telescope. Nature of Prominences and their Role in Space Weather. 2014, vol. 300, pp. 117-120. DOI: $10.1017 / \mathrm{S} 1743921313010831$.
Xue Z., Yan X., Cheng X., Yang L., Su Y., Kliem B., Zhang J., Liu Z., Bi Y., Xiang Y., Yang K., Zhao L. Observing the release of twist by magnetic reconnection in a solar filament eruption. Nature Communications. 2016, vol. 7, 11837. DOI: $10.1038 /$ ncomms 11837.

Yang S., Zhang J., Xiang Y. Magnetic Reconnection between small-scale loops observed with the New Vacuum Solar Telescope. Astrophys. J. Lett. 2015, vol. 798, iss. 1, L11, 6 p. DOI: 10.1088/2041-8205/798/1/L11.

Yang L., Peter H., He J.-S., Tu C.-Y., Wang L.-H., Zhang L., Yan L.-M. Formation of cool and warm jets by magnetic flux emerging from the solar chromosphere to transition region. Astrophys. J. 2018, vol. 852, iss. 1, article id. 16, 15 p. DOI: 10.3847/1538-4357/aa9996.

Young P.R., Tian H., Peter H., Rutten R.J., Nelson C.J., Huang Z., Schmieder B., Vissers G.J.M., Toriumi S., et al. Solar ultraviolet bursts. Space Sci. Rev. 2018, vol. 214, iss. 8, article id. 120, 39 p. DOI: 10.1007/s11214-018-0551-0.

Zhang M., Xia L.-D., Tian H., Chen Y. Signatures of transition region explosive events in hydrogen Ly $\beta$ profiles. Astron. Astrophys. 2010, vol. 520, article id. A37, 8 p. DOI: $10.1051 / 0004-6361 / 201014240$.

Zhang Y.Z., Shibata K., Wang J.X., Mao X.J., Matsumoto T., Liu Y., Su J.T. Revision of solar spicule classification. Astrophys. J. 2012, vol. 750, iss. 1, article id. 16, 9 p. DOI: 10.1088/0004$637 \mathrm{X} / 750 / 1 / 16$.

Zhao J., Schmieder B., Li H., Pariat E., Zhu X., Feng L., Grubecka M. Observational evidence of magnetic reconnection for brightenings and transition region arcades in IRIS observations. Astrophys. J. 2017, vol. 836, iss. 1, article id. 52, 13 p. DOI: $10.3847 / 1538-4357 / 836 / 1 / 52$.

Zwaan C. Elements and patterns in the solar magnetic field. Ann. Rev. Astron. Astrophys. 1987, vol. 25, pp. 83-111. DOI: 10.1146/annurev.aa.25.090187.000503.

How to cite this article:

Zhenghua Huang, Bo Li, Lidong Xia. Observations of small-scale energetic events in the solar transition region: explosive events, UV bursts, and network jets. Solar-Terrestrial Physics. 2019. Vol. 5. Iss. 2. P. 58-68. DOI: 10.12737/stp-51201909. 